\title{
Dietary sugar restriction reduces hepatic de novo lipogenesis in boys with fatty liver disease
}

\author{
Stephanie T. Chung ${ }^{1}$ and Sheela N. Magge ${ }^{2}$ \\ 'Diabetes, Endocrinology, and Obesity Branch, National Institutes of Health, Bethesda, Maryland, USA. Division of Pediatric Endocrinology and Diabetes, Johns Hopkins University School of Medicine, \\ Baltimore, Maryland, USA.
}

\begin{abstract}
Nonalcoholic fatty liver disease (NAFLD) in children resulting from the obesity epidemic is widespread and increasing. Although the complexities of pediatric NAFLD are recognized, screening and therapies in children remain limited. Moreover, pediatric NAFLD diagnosis fails to consider insulin resistance and metabolic dysfunction as important determinants. In this issue of the $J C l$, Cohen et al. explored the contribution of dietary factors to the pathogenesis of NAFLD in adolescent boys with biopsy-proven NAFLD and control participants. Notably, dietary sugar restriction over 8 weeks decreased de novo lipogenesis (DNL) and hepatic fat. The change in DNL correlated with changes in insulin and weight, but not with changes in hepatic fat, supporting the relevance of metabolic dysfunction to NAFLD. These results confirm the pathological link between excessive dietary sugar intake and NAFLD in children and support recent recommendations to change the nomenclature of NAFLD to metabolic associated fatty liver disease (MAFLD).
\end{abstract}

\section{Defining NAFLD in children}

Nonalcoholic fatty liver disease (NAFLD) is increasing in children worldwide as a result of the obesity epidemic and has emerged as an important risk factor for cardiometabolic disease $(1,2)$. Although the complications and burden of pediatric NAFLD are well recognized, there are critical barriers to widespread screening and effective therapies in children. The prevalence rates of NAFLD are highly variable and range from $5 \%$ to $10 \%$ in the general primary care setting to approximately $30 \%$ to $50 \%$ in specialty obesity clinics $(1,3,4)$. According to the guidelines of the North American Society for Pediatric Gastroenterology, Hepatology and Nutrition and the European Society for Pediatric Gastroenterology, Hepatology and Nutrition, NAFLD in children is a diagnosis of exclusion defined as chronic hepatic steatosis in children (18 years or younger) and exclusion of other causes of hepatic steatosis, such as genetic or metabolic disorders, infections, use of steatogenic medications, alcohol consumption, or malnutrition (5). NAFLD diagnostic procedures are invasive (e.g., liver biopsy), expensive, and complex, sometimes requiring sedation in children (e.g., magnetic resonant imaging), or are limited because of low biomarker sensitivity (e.g., alanine amino transaminase). Further, in contrast to established disease diagnoses, the definition of pediatric NAFLD is exclusionary and does not consider the roles of insulin resistance and metabolic dysfunction as important pathological determinants (5).

\section{Metabolic basis of NAFLD}

Recent recommendations by expert consensus panels suggest a change in the

\section{Related Article: https://doi.org/10.1172/JC1150996}

Conflict of interest: The authors have declared that no conflict of interest exists.

nomenclature of NAFLD to MAFLD (metabolic associated fatty liver disease) for adults and children $(6,7)$. The criteria for children are the presence of intrahepatic fat accumulation (steatosis based on liver histology, imaging, or blood biomarker) and one of three metabolic conditions: excess adiposity, presence of prediabetes or type 2 diabetes, or evidence of metabolic dysregulation (including dyslipidemia and hypertension) (6). The purpose of the revised definition is to align MAFLD diagnosis to treatment and prevention based on studies showing the importance of mitigating insulin resistance and metabolic disease (6). In this issue of the JCI, Cohen et al. provide timely insight highlighting the importance of modifiable dietary factors such as sugars in the pathogenesis of NAFLD in children (8).

Contemporary data confirm that NAFLD is a systemic metabolic disease involving impaired insulin signaling, overnutrition, and inflammation. Hepatic steatosis results from reductions in VLDL triglyceride export, accumulation of intrahepatic fat due to increased reesterification of free fatty acids (FFAs), and increased de novo lipogenesis (DNL) (9). DNL, the process by which enzymes convert nonlipid precursors into FFA, contributes $10 \%$ to $40 \%$ of FFA precursors to intrahepatic triglycerides. Overnutrition (including consuming excess dietary free sugars) stimulates DNL by increasing the availability of carbohydrate precursors and inducing insulin-mediated upregulation of lipogenic genes (SREBP-1c, FAS, $A C C, S C D 1$, and LPIN1) and enzymatic regulation of lipogenic pathways (10). The concept of selective insulin resistance is critical for understanding the metabolic effects of intrahepatic fat accumulation. Specifically, hyperinsulinemia within the liver promotes DNL, while peripheral insulin resistance in adipose tissue promotes lipolysis and the generation of FFAs that are subsequently transported to and 
reesterified in the liver (11). It is assumed that the mechanisms for NAFLD are similar in adults and children, but the exact pathological pathways, including the contributions of DNL and FFA reesterification to intrahepatic fat accumulation, are not established in children (12-14).

\section{DNL in pediatric NAFLD}

The study by Cohen et al. (8) confirms the pathological link between dietary free-sugar intake, DNL, and intrahepatic fat accumulation. A subgroup of 29 adolescent boys (ages 11 to 16 years old), out of a total of 40 boys, with biopsy-proven NAFLD were enrolled in an 8-week randomized controlled trial of dietary sugar restriction with less than $3 \%$ of calories from free sugar. Free sugar was defined as sugars added to foods and beverages as well as the sugar in fruit juice. Liver fat was measured using magnetic resonance imaging-proton density fat fraction (MRI-PDFF). The fractional contribution of hepatic DNL to triglycerides in palmitate was measured using deuterium-labeled water and mass isotopomer distribution analysis. Free-sugar restriction resulted in greater differences in free-sugar intake $(9.9 \%$ to $1.1 \%)$ compared with the usual-diet group (13.1\% to $11.8 \%$ ), a $10 \%$ reduction in DNL, an approximately $7 \%$ decrease in hepatic fat, and improvements in several metabolic parameters, including fasting insulin, glucose, triglycerides, LDL-C, and liver enzymes. These findings extend a previous short-term (9-day) study in youths demonstrating that dietary fructose restriction reduced DNL and liver fat (12). Together, these studies show that DNL is an important modifiable disease mechanism in NAFLD pathogenesis amenable to dietary free-sugar restriction strategies in children and adults. Using detailed and precise stable isotope methodology to quantify fractional DNL, the study (8) showed that a reduction in dietary free-sugar intake was associated with reductions in both DNL and percentage hepatic fat. However, the change in DNL correlated with changes in insulin and body weight, but not with change in hepatic fat. These findings indicate that while DNL is an important contributor to excess intrahepatic triglyceride accumulation, there is widespread metabolic dysregulation of intrahepatic fat accumulation that involves multiple pro- cesses, including defects in insulin signaling and overnutrition.

\section{Limitations and future directions}

Some important study limitations are worthy of consideration. Residual deuterium labeling during the 8-week period could result in overestimation of fractional DNL after the study intervention. To adjust for this residual labeling, a correction factor was derived. This empirical correction factor has not been validated and the effect size of dietary sugar restriction should be interpreted with caution. In addition, the imputation of DNL in 2 out of 13 (15\%) of the youths in the control group at 8 weeks could introduce bias by underestimating the change in DNL in this group. Of note, this study was limited to pubertal boys, mainly of Hispanic ethnicity; hence, further studies are needed to evaluate the intervention in larger multiethnic populations. Conducting prospective investigations, such as that by Cohen et al. (8), in multiethnic adolescent cohorts is critical because youths have unique age-related factors that could influence disease processes and interpretation. Adolescence is a time of physiological insulin resistance (15) and given the central role of insulin resistance in NAFLD development, it is important to assess whether the DNL and lipolysis/FFA release is amenable to dietary intervention. Further, hepatic fat accumulation and the contributions of DNL to this process vary widely among racial/ ethnic groups (16) and may be mediated by diet-gene interactions in key enzymes controlling DNL (e.g., stearoyl-CoA desaturases) (17). While reducing DNL may be an important component of treating fatty liver disease in boys of Hispanic ethnicity, DNL may not be the primary contributor to NAFLD development in youths of African ancestry. Holistic interventions that target the entire metabolic pathways of hepatic steatosis (DNL, FFA reesterification, and insulin resistance) are recommended. This latter point provides additional justification that a shift in terminology is warranted to foster research and clinical collaboration focusing on disease pathogenesis and treatment across the lifespan.

Address correspondence to: Sheela N. Magge, Division of Pediatric Endocrinology and Diabetes, Johns Hopkins
University School of Medicine, $200 \mathrm{~N}$. Wolfe Street, Rubenstein Bldg, Rm 3114, Baltimore, Maryland 21287, USA. Phone: 410.955.6463, option 2; Email: smagge3@jhmi.edu.

1. Anderson EL, et al. The prevalence of nonalcoholic fatty liver disease in children and adolescents: a systematic review and meta-analysis. PLoS One. 2015;10(10):e0140908.

2. Chung ST, et al. Cardiometabolic risk in obese children. Ann N Y Acad Sci. 2018;1411(1):166-183.

3. Zhou X, et al. [Prevalence of nonalcoholic fatty liver disease and metabolic abnormalities in 387 obese children and adolescents in Beijing, China]. Zhonghua Liu Xing Bing Xue Za Zhi. 2013;34(5):446-450.

4. Wiegand $S$, et al. Obese boys at increased risk for nonalcoholic liver disease: evaluation of 16,390 overweight or obese children and adolescents. Int JObes (Lond). 2010;34(10):1468-1474.

5. Vos MB, et al. NASPGHAN Clinical practice guideline for the diagnosis and treatment of nonalcoholic fatty liver disease in children: recommendations from the expert committee on NAFLD (ECON) and the North American Society of Pediatric Gastroenterology, Hepatology and Nutrition (NASPGHAN). J Pediatr Gastroenterol Nutr. 2017;64(2):319-334.

6. Eslam M, et al. Defining paediatric metabolic (dysfunction)-associated fatty liver disease: an international expert consensus statement. Lancet Gastroenterol Hepatol. 2021;6(10):864-873.

7. Eslam M, et al. A new definition for metabolic dysfunction-associated fatty liver disease: an international expert consensus statement. J Hepatol. 2020;73(1):202-209.

8. Cohen C, et al. Dietary sugar restriction reduces hepatic de novo lipogenesis in adolescent boys with fatty liver disease. JClin Invest. 2021;131(24):e150996.

9. Donnelly KL, et al. Sources of fatty acids stored in liver and secreted via lipoproteins in patients with nonalcoholic fatty liver disease. J Clin Invest. 2005;115(5):1343-1351.

10. Petersen MC, Shulman GI. Mechanisms of insulin action and insulin resistance. Physiol Rev. 2018;98(4):2133-2223.

11. Sekizkardes $\mathrm{H}$, et al. Free fatty acid processing diverges in human pathologic insulin resistance conditions. JClin Invest. 2020;130 (7):3592-3602.

12. Schwarz JM, et al. Effects of dietary fructose restriction on liver fat, de novo lipogenesis, and insulin kinetics in children with obesity. Gastroenterology. 2017;153(3):743-752.

13. Erkin-Cakmak A, et al. Isocaloric fructose restriction reduces serum D-lactate concentration in children with obesity and metabolic syndrome. $J$ Clin Endocrinol Metab. 2019;104(7):3003-3011.

14. Cioffi CE, et al. Hepatic fat is a stronger correlate of key clinical and molecular abnormalities than visceral and abdominal subcutaneous fat in youth. BMJ Open Diabetes Res Care. 2020;8(1):e001126.

15. Marwitz SE, et al. Cross-sectional and longitudinal examination of insulin sensitivity and secretion across puberty among non-Hispanic black 
and white children. Endocrinol Metab (Seoul). 2020;35(4):847-857.

16. Tricò D, et al. Intrahepatic fat, irrespective of ethnicity, is associated with reduced endogenous insulin clearance and hepatic insulin resistance in obese youths: a cross-sectional and longitudinal study from the Yale pediatric NAFLD cohort. Diabetes Obes Metab. 2020;22(9):1628-1638.
17. Chung ST, et al. Triglyceride paradox is related to lipoprotein size, visceral adiposity and stearoyl-CoA desaturase activity in black versus white women. Circ Res. 2020;126(1):94-108. 